\title{
Die Auswirkung von Digitalisierung auf Bildungs- und Sozialpolitik
}

\author{
Marius R. Busemeyer
}

\begin{abstract}
Zusammenfassung
In öffentlichen Debatten zu den Auswirkungen der digitalen Revolution auf die Arbeitsmärkte dominieren häufig pessimistische Zukunftsszenarien, die einen massiven Abbau von Beschäftigung und zunehmende Ungleichheit erwarten lassen. Im Gegensatz dazu gibt dieser Beitrag eine kurze Einführung in die sozialwissenschaftliche Forschung zu diesem Thema, die wesentlich differenziertere Befunde liefert. Ein weiterer Schwerpunkt dieses Kapitels ist die Diskussion der bildungs- und sozialpolitischen Reaktionen auf strukturelle Veränderungen im Zusammenhang mit der Digitalisierung. Hier werden unterschiedliche Policy-Lösungen wie Weiterbildung, lebenslanges Lernen, aktive Arbeitsmarktpolitik und das bedingungslose Grundeinkommen angesprochen. Das Kapitel schließt mit einer These zu den künftigen Entwicklungen, die besagt, dass politische Faktoren und Entscheidungen einen maßgeblichen Einfluss darauf haben werden, ob die Digitalisierung zu einer Verschärfung sozio-ökonomischer Ungleichheit beiträgt oder diese lindert.
\end{abstract}

\section{Schlüsselwörter}

Bildung · Sozialpolitik · Ungleich · Automatisierung · Policy-Analyse

Radikaler technologischer Wandel in Form von Digitalisierung und Automatisierung hat zunehmend Auswirkungen auf den Arbeitsmarkt und damit indirekt auf Bildungs- und Sozialpolitik. Die exponentielle Verbreitung neuer Technologien eröffnet einerseits neue Beschäftigungsmöglichkeiten und generiert wirtschaftlichen Wohlstand. Andererseits werden zunehmend Befürchtungen geäußert, dass der radikale technologische Wandel einen neuen massiven Rationalisierungsprozess befördern könnte, der mit dem Abbau von Arbeitsplätzen und der Prekarisierung von bestehenden Arbeitsverhältnissen einhergehen würde.

M. R. Busemeyer ( $\square)$

Fachbereich Politik- und Verwaltungswissenschaft, Universität Konstanz, Konstanz, Deutschland

E-Mail: Marius.Busemeyer@uni-konstanz.de 
Hier sollen die Konturen dieser noch am Anfang stehenden Debatten nachgezeichnet werden. Zunächst sind begrifflich und konzeptionell die unterschiedlichen Entwicklungen aufzuarbeiten, die in öffentlichen Diskussionen häufig mit dem Schlagwort „Digitalisierung“ der Arbeitswelt in Verbindung gebracht werden. Hierbei handelt es sich um durchaus unterschiedliche Phänomene - wie etwa das Aufkommen neuer Informations- und Kommunikationstechnologien, Fortschritte in der Robotik, Entwicklungen in der künstlichen Intelligenz, die Plattform-Ökonomie, das Internet der Dinge und neue Produktions- und Regelsysteme (,Industrie 4.0“) -, deren unterschiedliche Auswirkungen auf Arbeitsmärkte, Bildungs- und Sozialpolitik in wesentlich differenzierter Form diskutiert werden müssen als es teilweise heute geschieht.

Der zweite Abschnitt des Artikels gibt einen kurzen Einblick in die aktuelle Forschung zu den Auswirkungen von Digitalisierung und Automatisierung auf den Arbeitsmarkt. Auch wenn die Untersuchung von technologischem Wandel auf sozioökonomische Arbeitsmarkt-Outcomes ein klassisches Thema in der Literatur ist, ist die Zahl der Arbeiten, die sich mit den Konsequenzen der Digitalisierung und Automatisierung im engeren Sinne befassen, noch recht überschaubar. Im dritten Abschnitt werden dann die sozial- und bildungspolitischen Implikationen dieser Veränderungsprozesse diskutiert. Auch wenn die realen Auswirkungen von Digitalisierung und Automatisierung auf den Arbeitsmärkten schwer vorherzusagen sind, werden bereits heute und mit zunehmender Intensität politische Debatten über die notwendigen Policy-Reaktionen geführt. Von daher sollte sich die vergleichende Policy-Forschung in diesem Bereich jetzt schon der Analyse dieser Debatten widmen.

\section{Klärung des Begriffs}

Die Auswirkungen von technologischem Wandel im digitalen Zeitalter auf Wirtschaft, Politik und Gesellschaft sind vielfältig und multidimensional. Begriffe wie Digitalisierung, Automatisierung, „Industrie 4.0“, die Plattform- und „Sharing“Ökonomie bezeichnen Teilaspekte dieses umfassenden Veränderungsprozesses, ohne diesen in seiner Gesamtheit erfassen zu können. Die weiteren Ausführungen konzentrieren sich auf die durch Rationalisierung und Umorganisation von Arbeitsprozessen oder Umverteilung von Arbeit direkt bewirkten Effekte auf den Arbeitsmarkt.

Hierbei werden drei Aspekte des technologischen Wandels in den Vordergrund gestellt:

(1) Die fortschreitende Entwicklung von Informations- und Kommunikationstechnologien (ICT), die durch die exponentielle Vergrößerung von Datenverarbeitungs- und -speicherkapazitäten (,Big Data“) massive Auswirkungen auf die Organisation von Arbeitsprozessen haben wird;

(2) neue Möglichkeiten zur Automatisierung von manuellen Fertigungsprozessen, die durch das Zusammenwirken von „Big Data“, künstlicher Intelligenz und dem Internet der Dinge entstehen; sowie

(3) das Aufkommen der Plattform- oder "Sharing"-Ökonomie, die neue Formen projektorientierter und -zentrierter Zusammenarbeit ermöglicht (daher ist in diesem Kontext auch oft der Begriff der ,Gig-Economy“ zu hören, der auf die Kurzfristigkeit der Arbeitsprojekte verweist). 
Diese drei Aspekte technologischen Wandels schließen sich natürlich nicht gegenseitig aus, sondern verstärken sich wechselseitig. Auch sind die begrifflichen Abgrenzungen der einzelnen Dimensionen nicht immer eindeutig möglich. Wechselseitige Komplementaritäten bestehen beispielsweise zwischen „Big Data“ und künstlicher Intelligenz („Machine Learning“) sowie zwischen dem Aufkommen neuer Informations- und Kommunikationstechnologien (insbesondere den sozialen Medien) und der Plattform-Ökonomie. Diese funktionalen Komplementaritäten zwischen den unterschiedlichen Dimensionen sind - so könnte man argumentieren - ein wesentlicher Grund dafür, dass sich die aktuelle Phase technologischen Wandels qualitativ von früheren Phasen unterscheiden könnte, denn die Gleichzeitigkeit radikaler technologischer Veränderungen in verschiedenen Bereichen befördert selbst wiederum exponentiell verlaufende Wachstums- und Veränderungsprozesse.

Abgesehen von den Auswirkungen der digitalen Revolution auf den Arbeitsmarkt, um die es hier primär gehen soll, hat die Digitalisierung auch Konsequenzen für die Organisation und Erbringung von wohlfahrtsstaatlichen Dienstleistungen selbst. Es ist z. B. zu erwarten, dass die Digitalisierung von gesundheitsrelevanten Daten die Finanzierung und Erbringung von Gesundheitsdiensten verändern wird. Die Digitalisierung von Gesundheitsdaten ermöglicht dabei einerseits personalisierte Dienstleistungen und eine bessere Vernetzung zwischen Gesundheitsanbietern, kann aber andererseits dazu führen, dass Krankenversicherungen ihre Risikokalkulationen sehr viel stärker auf individuelle Risiken hin zuschneiden können und damit Diskussionen um risikoabhängige Versicherungsprämien befördert werden. Im Bildungsbereich wird das Schlagwort der Digitalisierung häufig mit der Einführung von neuen, technologiezentrierten Lehr- und Lernmitteln gleichgesetzt. Auch die Einführung neuer Lerninhalte - wie etwa die Vermittlung von Programmierkenntnissen im Rahmen des Schulunterrichts - wird diskutiert.

Theoretische Grundlagen: Überblick über den Stand der Forschung zu Auswirkungen von Digitalisierung und Automatisierung

Die Auswirkungen der oben angesprochenen technologischen Veränderungsprozesse auf den Arbeitsmarkt sind ambivalent. In den öffentlichen Diskussionen zu diesen Themen dominieren häufig die pessimistischen Zukunftsszenarien, die den Wegfall einer großen Zahl von Arbeitsplätzen vorhersagen. Es gibt jedoch auch gute Gründe dafür anzunehmen, dass radikale technologische Veränderungen neue Beschäftigungspotenziale erschließen. Zwar hat die Verbreitung von neuen ICT und des Internets viele etablierte Branchen unter Druck gesetzt, gleichzeitig sind allerdings neue Beschäftigungspotenziale und gänzlich neue Berufs- und Beschäftigungskategorien (wie etwa Blogger oder You Tube „Influencer“) entstanden. Der Zugang zu vormals elitär geschlossenen Märkten für Musik, Film und Publizistik hat sich geöffnet. In gleicher Weise hat das Aufkommen von Unternehmen der PlattformÖkonomie (AirBnB für Übernachtungsmöglichkeiten, Uber und Lyft für Taxifahrten, aber auch Plattformen für generische, selbstständige Dienstleistungen wie TaskRabbit oder Amazons MTurk) einerseits vormals geschlossene Märkte geöffnet, andererseits auch zu einer Verbreitung von prekären Beschäftigungsverhältnissen 
und der Unterminierung von etablierten Formen der Arbeitsmarktregulierung beigetragen (Eichhorst et al. 2017).

In ähnlicher Weise hat die fortschreitende Automatisierung und Robotisierung der Arbeitswelt zunächst Beschäftigungspotenziale rationalisiert und eliminiert. Durch die zu erwartende fortschreitende Entwicklung von Künstlicher Intelligenz und „Machine Learning“ werden weitere, auch anspruchsvollere Tätigkeiten automatisierbar: Selbst-lernende Software-Algorithmen können zunehmend RoutineTätigkeiten in sogenannten ,White-Collar-Berufen“ übernehmen (z. B. Buchhaltung, Steuerberatung oder Prüfung von Rechtsfragen, vgl. Autor et al. 2003). Aus einer pessimistischen Perspektive betrachtet könnte radikaler technologischer Wandel somit zum Wegfall einer hohen Zahl von Arbeitsplätzen führen. Die optimistische Gegenperspektive hingegen besteht darin, dass der Wegfall von Routine-Tätigkeiten in Fertigungs- und einigen Dienstleistungsberufen insgesamt zu einer Verbesserung der Beschäftigungsbedingungen beitragen könnte, wenn tendenziell anspruchsvollere und qualitativ hochwertige Beschäftigungsmöglichkeiten übrig bleiben. Welche dieser beiden Perspektiven die zutreffendere ist, hängt maßgeblich davon $a b$, ob es gelingt, diejenigen, deren Arbeitsplatz durch technologisch bedingte Rationalisierung wegfällt, durch Weiterbildung und aktive Arbeitsmarktpolitik in die Lage zu versetzen, auch anderen Tätigkeiten nachzugehen.

Die aktuelle Forschung zu den Auswirkungen der digitalen Revolution auf den Arbeitsmarkt hat noch keine abschließende Antwort zu der Frage gefunden, wie umfassend die Veränderungen bereits sind oder in naher Zukunft sein werden. Die Forschung steht hierbei vor dem grundlegenden Problem, dass die gegenwärtigen Auswirkungen des mit der Digitalisierung einhergehenden technologischen Wandels auf den Arbeitsmarkt noch begrenzt sind, aufgrund der oben angesprochenen, sich selbst verstärkenden Dynamik aber in naher Zukunft durchaus dramatischere Dimensionen annehmen könnten. Daher kann die einschlägige Forschung in zwei Schwerpunkte unterteilt werden.

Zum einen sind dies Studien, die die Auswirkungen von technologischem Wandel und Internationalisierung (Globalisierung) auf Arbeitsmarkt-Outcomes wie Beschäftigung und Ungleichheit mit bereits erhobenen Daten retrospektiv für die Vergangenheit untersuchen. Aufbauend auf den grundlegenden Arbeiten von Autor et al. (2003) untersucht diese Forschungsrichtung, inwiefern technologischer Wandel zu einer Polarisierung von Beschäftigungsmöglichkeiten - und damit langfristig auch von Löhnen - beigetragen hat. Viele Studien (vgl. zum Beispiel Goos et al. 2014 für ein größeres Sample von OECD-Staaten) bestätigen die These von der zunehmenden Polarisierung von Arbeitsmarktstrukturen: Im Kern zeigen diese Arbeiten, dass technologischer Wandel nicht einfach zu einer Zunahme von Ungleichheit führt. Stattdessen kommt es zu einem „Aushöhlungsprozess“ der Mittelschichten: Nachdem einfache, Routine-basierte Tätigkeiten bereits in vorangehenden Wellen technologischer Rationalisierung weggefallen sind, seien nun anspruchsvollere Routine-Tätigkeiten in typischen Mittelklasse-Berufen am stärksten von technologischen Veränderungen betroffen. Relativ geschützt vor Veränderungen seien hoch anspruchsvolle, kreative und soziale Tätigkeiten am oberen Ende der Einkommens- und Bildungsskala sowie einfache, nicht-routinisierbare Tätigkei- 
ten am unteren Ende dieser Skalen, vor allen personenbezogene Dienstleistungen. Wenn es somit den Angehörigen der Mittelschicht nicht gelingt, anspruchsvolle Berufe am oberen Ende der Einkommens- und Bildungsskala zu erreichen, droht der relative Absturz in die unteren Einkommensschichten. Auch wenn die Evidenz für den Zusammenhang zwischen technologischem Wandel und Arbeitsmarktpolarisierung zunehmend robuster wird, laufen die Veränderungsprozesse, die in diesen Studien beschrieben werden, eher graduell ab und sind schwerlich mit den dramatischen Prognosen der Zukunftspessimisten in Einklang zu bringen, zumal viele OECD-Länder gegenwärtig historisch niedrige Arbeitslosenraten aufweisen.

Daher befasst sich eine zweite Gruppe von Studien mit den zukünftigen Auswirkungen von technologischem Wandel. Besonders einflussreich in diesem Zusammenhang ist die Studie von Frey und Osborne (2017), die bereits einige Jahre zuvor als Arbeitspapier veröffentlicht wurde und für ein großes Medienecho sorgte. Die zentrale methodische und empirische Herausforderung für Studien dieser Art ist, dass aus offensichtlichen Gründen noch keine realen Arbeitsmarktdaten vorliegen. Frey und Osborne (2017) begegnen dieser Herausforderung, indem sie eine Gruppe von Technologie-Experten zu ihren Einschätzungen hinsichtlich des Automatisierungspotenzials verschiedener, ausgewählter Tätigkeiten und Berufe befragen und diese Daten verwenden, um mit Hilfe von statistischen Methoden eine Einschätzung hinsichtlich des Automatisierungspotenzials aller Berufe abgeben zu können. Laut ihrer Schätzung sind 47 Prozent aller Beschäftigungsverhältnisse in den USA in Berufen einem hohen Automatisierungsrisiko (über 70-prozentige Wahrscheinlichkeit der Automatisierung in den nächsten Jahren) ausgesetzt. Nach den Schätzungen von Frey und Osborne (2017) sind besonders auch Mittelklasse-Berufe mit stark ausgeprägtem Routine-Charakter von der Automatisierung betroffen (z. B. Berufe in den Bereichen Buchhaltung, Dateneingabe, Verwaltung und Bankdienstleistungen), während Berufe in den sozialen, persönlichen und kreativen Dienstleistungsbereichen einem geringen Automatisierungsrisiko ausgesetzt sind.

Die Studie von Frey und Osborne (2017) ist vielfach kritisiert worden und erkennt auch selbst einige Einschränkungen ihrer Methodik an. So sagt die Studie nichts darüber aus, inwiefern der Wegfall von Beschäftigung durch Digitalisierung und Automatisierung durch die Entstehung neuer Beschäftigungsformen kompensiert werden kann. Voraussagen hinsichtlich neuer Berufsfelder und Beschäftigungsformen sind naturgemäß noch schwieriger als Aussagen hinsichtlich des Automatisierungspotenzials bestehender Berufe. Des Weiteren ist technologischer Wandel keine rein exogene Größe, sondern kann durch politisches Handeln beschleunigt (z. B. durch öffentliche Investitionen in Forschung und Entwicklung) oder ausgebremst werden. Schließlich kann die Tatsache, dass Frey und Osborne (2017) Technologie-Experten befragten, dazu beigetragen haben, dass Automatisierungspotenziale systematisch überschätzt werden.

Eine weitere, eher methodische Kritik bezieht sich darauf, dass Frey und Osborne (2017) Automatisierungspotenziale auf Grundlage ganzer Berufe abschätzen und nicht einzelne Tätigkeiten (,tasks“) betrachten. Eine konsequent „task-zentrierte“ Perspektive (Autor et al. 2003) hingegen zeigt, dass zwar einzelne Tätigkeiten ein hohes Automatisierungspotenzial aufweisen, dies aber nur bedingt einen Beruf (als 
„Bündel“ unterschiedlicher Tätigkeiten) als Ganzes betreffen muss. Die Automatisierung einzelner Tätigkeiten innerhalb eines Berufes würde dann zu einer Verschiebung der relativen Bedeutung dieser Tätigkeiten führen, aber nicht zwangsläufig den Beruf insgesamt eliminieren.

Studien, die eine konsequent ,task-zentrierte“ Perspektive einnehmen, kommen zu deutlich niedrigeren Zahlen hinsichtlich der zu erwartenden Veränderungen auf dem Arbeitsmarkt. Bonin et al. (2015) übertragen den Ansatz von Frey und Osborne (2017) auf Deutschland und kommen bei gleicher Methodik auf eine ähnlich hohe Zahl (42 Prozent der Beschäftigungsverhältnisse in Berufen mit einer hohen Automatisierungswahrscheinlichkeit in den nächsten Jahren). Wenn man nun allerdings von einer Berufe- auf eine ,task“-zentrierte Perspektive wechselt, schätzen Bonin et al. (2015), dass nur etwa 9 Prozent der Beschäftigungsverhältnisse einem hohen Automatisierungsrisiko ausgesetzt sind (12 Prozent für den Fall USA). Arntz et al. (2016) führen eine Analyse für ein größeres Sample von OECD-Staaten durch und kommen zu ähnlichen Zahlen: Im Durchschnitt von 21 OECD-Staaten seien 9 Prozent der Beschäftigungsverhältnisse einem hohen Automatisierungsrisiko ausgesetzt mit einer Spannweite von 6 Prozent in Korea bis hin zu 12 Prozent in Österreich.

Zusammenfassend kann somit festgehalten werden, dass bisher noch kein Konsens in der Literatur hinsichtlich der gegenwärtigen und in naher Zukunft zu erwartenden Auswirkungen der Digitalisierung und Automatisierung auf Arbeitsmärkte existiert. Es ist auch nicht zu erwarten, dass ein solcher Konsens kurzfristig erreichbar ist. Die unterschiedlichen Studien, die hier kurz vorgestellt wurden, nehmen unterschiedliche Analyseperspektiven ein, und die damit einhergehenden methodologischen Entscheidungen sind durchaus nachvollziehbar und haben ihre jeweilige Berechtigung. Trotz des fehlenden Konsenses hinsichtlich der Größe des Effekts (von „dramatisch“ bis ,signifikant“) verbindet die hier vorgestellten Studien jedoch die Einsicht, dass technologischer Wandel sowohl in der Vergangenheit als auch in der Gegenwart in nicht-trivialer Weise Arbeitsmarktstrukturen und -prozesse beeinflusst.

\section{Praktische Anwendungen und Implikationen für die Bildungs- und Sozialpoli- tik}

Eine weitere Gemeinsamkeit der im vorherigen Abschnitt vorgestellten Studien besteht darin, dass sie sich mit den direkten Auswirkungen von Digitalisierung und Automatisierung auf den Arbeitsmarkt befassen, nicht aber mit den sozialund bildungspolitischen Folgen dieser Entwicklungen. Bislang gibt es nur eine Handvoll von Arbeiten, die sich diesem Thema widmen. Thewissen und Rueda (2019) beispielsweise untersuchen unter Verwendung der in der oben skizzierten ökonomischen Literatur entwickelten Maße und Indikatoren, inwiefern das individuelle Automatisierungsrisiko eines Berufs mit Unterstützung für staatliche Umverteilungsmaßnahmen einhergeht und finden einen positiven Zusammenhang. Eichhorst et al. (2017) beschäftigen sich mit den Auswirkungen der Prekarisierung von Beschäftigungsverhältnissen in der „Gig“- bzw. Plattform-Ökonomie auf Arbeitsmarktregulation. 
Auch wenn detaillierte wissenschaftliche Untersuchungen zu den bildungs- und sozialpolitischen Reaktionen noch weitestgehend ausstehen, gibt es durchaus bereits Debatten über die relativen Vor- und Nachteile unterschiedlicher Policy-Lösungen. Eine erste und besonders naheliegende Policy-Empfehlung besteht darin, öffentliche und private Investitionen in Bildung auszuweiten und insbesondere berufliche Weiterbildung, lebenslanges Lernen und aktive Arbeitsmarkpolitik zu fördern (Colin and Palier 2015). Da hoch qualifizierte Berufe und Tätigkeiten vor den Auswirkungen der Digitalisierung und Automatisierung besser geschützt sind als Routinebasierte, einfachere Tätigkeiten, könnten Investitionen in Bildung sowohl auf individueller als auch auf gesellschaftlicher Ebene einen effektiven Schutz gegen Rationalisierungsmaßnahmen darstellen.

Die Diskussion der Rolle der Bildungspolitik im Prozess der Digitalisierung hat unterschiedliche Ebenen. Zum einen geht es um die Verbesserung des Zugangs zu weiterführenden Bildungsmöglichkeiten für Berufstätige. Der Ausbau von finanzieller Unterstützung für individuelle Bildungsmaßnahmen, beispielsweise über Bildungsgutscheine oder Bildungskonten (wie im Mai 2017 von der ehemaligen Bundesarbeitsministerin Andrea Nahles vorgeschlagen), könnte Beschäftige bei der Erschließung neuer Beschäftigungsoptionen unterstützen. Diese Maßnahmen könnten durch gesetzliche oder tarifvertragliche Regelungen zur Freistellung von Arbeitnehmer*innen für kurzfristige und längere Weiterbildungsmaßnahmen („Sabbatical“ und „Recht auf Weiterbildung“) flankiert werden. So gibt es in Österreich schon seit längerem das Instrument der „Bildungskarenz“, das Arbeitnehmer*innen erlaubt, mit finanzieller Absicherung durch die Arbeitslosenversicherung eine Weiterbildungsmaßnahme von bis zu einem Jahr umzusetzen.

Maßnahmen, die auf den Ausbau von Weiterbildung und lebenslangem Lernen abzielen, stellen eine naheliegende Antwort auf die Herausforderung der Digitalisierung dar, haben allerdings auch inhärente Grenzen. So hängt die Wirksamkeit von Weiterbildungsmaßnahmen letztlich davon ab, dass tatsächlich genügend neue Beschäftigungsoptionen in neuen Wirtschaftsbereichen entstehen, um rein quantitativ den Wegfall traditioneller Beschäftigung zu kompensieren. Um eine Zunahme von Arbeitsmarktstratifizierung zu vermeiden, müssten die neuen Beschäftigungsmöglichkeiten auch qualitativ gleich- oder höherwertiger sein. Und schließlich ist keineswegs garantiert, dass diejenigen, deren Arbeitsplatz der Rationalisierung zum Opfer fällt, die notwendigen grundlegenden kognitiven und sozialen Voraussetzungen mitbringen, um erfolgreich eine Weiterbildung zu absolvieren. Wenn Tätigkeiten und Berufe im Zeitalter der Digitalisierung immer anspruchsvoller werden, könnte es sein, dass ein gewisser und wachsender Teil der Arbeitnehmerschaft trotz Weiterbildung nicht in der Lage ist, diese Tätigkeiten auszuüben.

Auch aus diesem Grund - und dies ist die zweite Ebene der bildungspolitischen Diskussion - werden zunehmend grundlegende Reformen der Bildungssysteme selbst gefördert. Ein Aspekt in dieser Debatte ist der Einsatz von technologiebasierten Lehr- und Lernmitteln, wobei es weiterhin empirisch umstritten ist, ob digitale Lehr- und Lernmethoden den traditionellen tatsächlich überlegen sind (Falck et al. 2018). Ein zweiter Aspekt betrifft die Reform von Lehrplänen und Lehrinhalten. In diesem Zusammenhang betont die Politik vor allen Dingen die Notwendigkeit 
der gezielten Förderung der sogenannten MINT-Fächer (Mathematik, Informatik, Naturwissenschaft und Technik). Der offensichtliche Zusammenhang zum Thema Digitalisierung besteht darin, dass erwartet wird, dass neue Beschäftigungspotenziale vor allen Dingen in den neuen Wirtschaftssektoren der digitalen Ökonomie entstehen. Daher - so die Erwartung - stellt eine frühzeitige Vorbereitung und Ausbildung der jüngeren Generationen durch den Ausbau der MINT-Fächer in Schulen und der Ausbildung eine besonders effektive Strategie zur Sicherung des Fachkräftenachwuchses dar. Auch wenn gegenwärtig ein Fachkräftemangel in Technik-basierten Berufen zu konstatieren ist, ist langfristig allerdings nur bedingt vorhersehbar, in welchen Bereichen der Ökonomie die größten Wachstumspotenziale bestehen. So zeichnet sich ebenfalls jetzt bereits ein Fachkräftemangel in den sozialen Dienstleistungs- und Pflegeberufen ab, die laut den oben zitierten Studien ebenfalls recht weitgehend vor Digitalisierung und Automatisierung geschützt sind, da sie auf sozialen und interpersonalen Fähigkeiten aufbauen. Gleiches gilt für Tätigkeiten in der Kreativwirtschaft. Insofern ist abzuwarten, ob der Diskurs um die Neuausrichtung von Lehrplänen und Lehrinhalten mittelfristig auch stärker auf die Vermittlung von sozialen, kulturellen und kreativen Fertigkeiten hin ausgerichtet wird oder ob die Digitalisierungs-Diskussion einseitig die Förderung von MINTFächern privilegiert.

In den sozialpolitischen Diskussionen zu den Auswirkungen von Digitalisierung stehen arbeitsmarktpolitische Fragen im Vordergrund. Neben den oben bereits angesprochenen Vorschlägen zum Ausbau von Weiterbildung und lebenslangem Lernen geht es hierbei vor allen Dingen um die aktive Arbeitsmarktpolitik und in weitergehenden Vorschlägen - die Einführung eines bedingungslosen Grundeinkommens. Unter den Begriff der aktiven Arbeitsmarktpolitik kann ein breites Maßnahmenbündel gefasst werden, das neben Weiterbildungsmaßnahmen auch die Subventionen von öffentlichen Beschäftigungsmaßnahmen umfasst. Auch Kombinationen unterschiedliche Instrumente sind denkbar, etwa wenn ein Grundeinkommen nur für einen begrenzten Zeitraum gewährt wird und diese Zeit für Weiterbildungsmaßnahmen genutzt werden kann („Bildungskarenz“). In eine ähnliche Richtung geht die Idee eines „Partizipation income“, die der Ökonom Tony Atkinson (Atkinson 2015) vorgeschlagen hat. Atkinsons Idee besteht darin, dass der Staat eine Art Grundeinkommen auszahlt, diese Auszahlung aber davon abhängig macht, dass die Nutznießer sich in irgendeiner Form - sei es durch soziales Engagement im Non-Profit-Sektor, Weiterbildung, regulärer oder subventionierter Beschäftigung an der Gesellschaft beteiligen.

Im Gegensatz dazu lehnen weitergehende Vorschläge zur Einführung eines bedingungslosen Grundeinkommens (BGE) die Verknüpfung der Auszahlung des Grundeinkommens an Bedingungen grundsätzlich ab. Die Debatte zum BGE ist zu vielschichtig und komplex, um sie hier ausführlich darzustellen. Politisch interessant ist allerdings die Tatsache, dass das BGE von sehr unterschiedlichen politischen Akteuren unterstützt wird: Konservative und Libertäre erhoffen sich vom BGE eine Überwindung der Abhängigkeit vom traditionellen Sozialstaat, da alle bestehenden Sozialleistungen durch ein universales Grundeinkommen ersetzt werden könnten. Linke wiederum verbinden mit der Einführung eines BGE das Ende der Kommodi- 
fizierung der Arbeitskraft. Letztlich unterscheiden sich die Konzepte vom BGE grundlegend darin, wie großzügig das BGE ausgestaltet werden soll, und ob es mit einer Abschaffung bestehender Sozialleistungen einhergehen soll oder ob viele der etablierten sozialstaatlichen Leistungen weiterhin bestehen bleiben. Die Debatte um die Einführung eines BGE hat im Kontext der Digitalisierungs-Diskussion neuen Auftrieb erhalten, da ein BGE Sorgen um Massenarbeitslosigkeit und prekäre Beschäftigung in der digitalen Ökonomie lindern würde. Kritiker des BGE befürchten, dass es zu einem allgemeinen Rückgang der Erwerbsbeteiligung und der Beschäftigungsfähigkeit der Arbeitnehmerschaft beitragen könnte und davon bestimmte, bereits jetzt benachteiligte Bevölkerungsgruppen wie Frauen und Migranten besonders betroffen wären (Colin und Palier 2015).

\begin{abstract}
Ausblick: Künftige Entwicklung
Dieses kurze Kapitel konnte nur einen skizzenhaften Einblick in die Diskussion zu den bildungs- und sozialpolitischen Folgewirkungen der Digitalisierung und Automatisierung geben. Ohne Zweifel besteht weiterhin großer Forschungsbedarf, insbesondere hinsichtlich der Policy-Reaktionen auf die sich abzeichnenden strukturellen Verschiebungen auf den Arbeitsmärkten. Ob die digitale Revolution langfristig Ungleichheits-verstärkende Auswirkungen oder durch die Schaffung von neuen Beschäftigungsmöglichkeiten und Beteiligungsformen eine egalisierende Wirkung hat, hängt maßgeblich von politischen Faktoren und Entscheidungen ab. Bildungs- und Sozialpolitik werden hier eine zentrale Rolle spielen, aber welche Policy-Lösungen sich letztlich durchsetzen werden, ist heute nur in Grundrissen erkennbar.
\end{abstract}

\title{
Literatur
}

Arntz, Melanie, Terry Gregory, und Ulrich Zierahn. 2016. The risk of automation for Jobs in OECD Countries. A comparative analysis. (OECD social, employment and migration Working Papers 189). Paris: OECD Publishing.

Atkinson, Anthony B. 2015. Inequality. What can be done? Cambridge, MA: Harvard University Press.

Autor, David H., Frank Levy, und Richard J. Murnane. 2003. The skill content of recent technological change. An empirical exploration. The Quarterly Journal of Economics 118(4): 1279-1333.

Bonin, Holger, Terry Gregory, und Ulrich Zierahn. 2015. Übertragung der Studie von Frey/ Osborne (2013) auf Deutschland, Kurzexpertise Nr. 57. Mannheim: Zentrum für Europäische Wirtschaftsforschung.

Colin, Nicolas, und Bruno Palier. 2015. The next safety net social policy for a digital age. Foreign Affairs 94(4): 29-33.

Eichhorst, Werner, Holger Hinte, Ulf Rinne, und Verena Tobsch. 2017. How big is the gig? Assessing the preliminary evidence on the effects of digitalization on the labor market. Management Revue 28(3): 298-318.

Falck, Oliver, Constantin Mang, und Ludger Woessmann. 2018. Virtually no effect? Different uses of classroom computers and their effect on student achievement. Oxford Bulletin of Economics and Statistics 80(1): 1-38. 
Frey, Carl Benedikt, und Osborne, Michael A. 2017. The future of employment. How susceptible are jobs to computerisation? Technological Forecasting and Social Change 114(Issue C): 254-280.

Goos, Maarten, Alan Manning, und Anna Salomons. 2014. Explaining Job polarization: Routinebased technological change and offshoring. American Economic Review 104(8): 2509-2526.

Thewissen, Stefan, und David Rueda. 2019. Automation and the welfare State. Technological change as a determinant of redistribution preferences. Comparative Political Studies 52(2): 171-208. 